\title{
Criminologie
}

\section{Les frontières " ethniques " au sein de la police}

\section{Mylène Jaccoud}

Volume 36, numéro 2, automne 2003

Le construit de l'ethnicité en criminologie

URI : https://id.erudit.org/iderudit/007867ar

DOI : https://doi.org/10.7202/007867ar

Aller au sommaire du numéro

Éditeur(s)

Les Presses de l'Université de Montréal

ISSN

0316-0041 (imprimé)

1492-1367 (numérique)

Découvrir la revue

Citer cet article

Jaccoud, M. (2003). Les frontières « ethniques » au sein de la police.

Criminologie, 36(2), 69-87. https://doi.org/10.7202/007867ar

\section{Résumé de l'article}

Les " programmes d'accès à l'égalité » ont été mis en place dans les services de police au Canada en vue de favoriser l'insertion de groupes définis dans les catégories officielles comme appartenant, entre autres, à des groupes " ethnoculturels » et des " minorités visibles ». Dans quelle mesure ces programmes facilitent-ils l'insertion ? Quel est l'impact de ces programmes sur les rapports entre policiers issus du groupe majoritaire et policiers issus des groupes ciblés par ces programmes ? Ces programmes ont-ils pour effet de déconstruire, de recomposer ou de renforcer les frontières ethniques? L'impact de la présence de groupes minoritaires ou minorisés dans les services de police est analysé à partir des récits d'expérience de 47 policiers et policières oeuvrant au sein du Service de police de la Ville de Montréal.
Ce document est protégé par la loi sur le droit d'auteur. L'utilisation des services d'Érudit (y compris la reproduction) est assujettie à sa politique d'utilisation que vous pouvez consulter en ligne.

https://apropos.erudit.org/fr/usagers/politique-dutilisation/ 


\title{
Les frontières « ethniques » au sein de la police
}

\author{
Mylène Jaccoud \\ Professeure/chercheure \\ École de criminologie \\ Centre international de criminologie comparée \\ Université de Montréal \\ mylene.jaccoud@umontreal.ca
}

\begin{abstract}
Résumé • Les «programmes d'accès à l'égalité » ont été mis en place dans les services de police au Canada en vue de favoriser l'insertion de groupes définis dans les catégories officielles comme appartenant, entre autres, à des groupes «ethnoculturels» et des «minorités visibles». Dans quelle mesure ces programmes facilitent-ils l'insertion? Quel est l'impact de ces programmes sur les rapports entre policiers issus du groupe majoritaire et policiers issus des groupes ciblés par ces programmes? Ces programmes ont-ils pour effet de déconstruire, de recomposer ou de renforcer les frontières ethniques? L'impact de la présence de groupes minoritaires ou minorisés dans les services de police est analysé à partir des récits d'expérience de 47 policiers et policières œuvrant au sein du Service de police de la Ville de Montréal.
\end{abstract}

\begin{abstract}
The equity access programs were implemented within police departments throughout Canada with the aim of encouraging the inclusion of groups defined as ethnic and visible minorities. To what extent do they facilitate inclusion? What is the impact of these programs on the quality of the relationships between police officers from the majority group and minority police officers? Do these program deconstruct, transform or reinforce ethnic boundaries? The impact of the presence of minority groups within police services is analyzed from the experiences of 47 police officers from the Montreal Police Service.
\end{abstract}

\section{Les politiques d'accès à l'égalité dans les services policiers au Canada et au Québec}

La politique d'action positive déployée en Amérique du Nord à la fin des années 1960 en vue d'intégrer des policiers issus de minorités ethniques visait non seulement à répondre aux revendications politiques pour l'égalité des droits entre les citoyens mais encore à améliorer les rapports 
entre la police et les minorités racialisées et à diminuer ou prévenir le racisme présumé de la police. Au Canada, ce projet débute en 1970 dans un contexte où les frontières de l'État-nation se voient fragilisées par les revendications des nationalismes autochtone et québécois, mais aussi par les changements des profils migratoires. En effet, à partir des années 1970, l'immigration d'origine européenne décroît au profit d'une immigration provenant des pays tiers-mondistes. Ce contexte incite le gouvernement fédéral à proposer une nouvelle vision des rapports entre l'État et les groupes minoritaires par l'intermédiaire d'une politique de multiculturalisme. La Loi sur le multiculturalisme entre en vigueur en 1971 et institutionnalise désormais le principe de la diversité culturelle à titre de principe fondateur de l'État canadien. C'est dans ce contexte que la question de l'insertion des membres issus de diverses communautés culturelles dans les forces de l'ordre est soulevée.

Les politiques d'embauche des groupes racialisés dans les services de police ne s'inscrivent pas uniquement dans un registre de dénonciation de la discrimination systémique ou dans celui du redressement des iniquités structurelles. Elles s'insèrent aussi dans une volonté d'atténuer les tensions raciales et de renforcer l'efficacité du travail policier dans une société où le caractère ethnique ou multiculturel est reconnu (Jaccoud et Felices, 1999). L'inclusion des minoritaires dans la police est recommandée, entre autres, sur la foi qu'un policier issu de la même communauté qu'il dessert, c'est-à-dire qu' il contrôle, pourra, par ses «attributs», mieux infiltrer «sa» communauté et contribuer ainsi à l'accroissement du rendement et de l'efficacité de l'organisation pour laquelle il travaille (Jaccoud et Félices, 1999). Par conséquent, il ne s'agit pas seulement de redéfinir l'Étatnation, mais également de maintenir un certain contrôle sur les conséquences sociostructurelles (en matière de conflits sociaux) de la présence des populations immigrantes, que Leman (1994) nomme la «troisième force nationale», dans le giron national. Certains auteurs vont même associer les pratiques d'inclusion des groupes minoritaires dans les institutions étatiques aux pratiques de pacification introduites par les colonisateurs dans les colonies anglo-saxonnes en vue d'instaurer et maintenir le pouvoir de la métropole ${ }^{1}$.

À la suite de l'enchâssement des principes d'équité et d'égalité dans les chartes et législations, des programmes d'accès à l'égalité (PAE) en emploi

1. Voir en particulier l'analyse de Haveman (1988) sur la politique de recrutement des Autochtones dans les institutions de contrôle sociopénal au Canada. 
se mettent en place ${ }^{2}$. En 1991, le Service de police de la Communauté urbaine de Montréal, conformément à l'article 86.1 de la Charte québécoise de droits et libertés de la personne, lance officiellement son programme d'accès dans le «but primordial d'instaurer une représentation équitable des groupes visés, soit : les minorités visibles, les communautés ethnoculturelles, les Autochtones et les femmes». Le principe de la représentativité proportionnelle des groupes visés par le programme d'accès à l'égalité constitue le principe directeur de ce programme, principe selon lequel les groupes visés doivent être "présents au sein des effectifs et ce suivant la même proportion qu'ils ont au sein de la main-d'œuvre disponible sur le territoire de la CUM» (SPCUM, $1997: 2$ ).

\section{Les notions de minorités visibles et de communautés ethnoculturelles}

Au Canada, les minorités visibles sont définies en vertu de la Loi sur l'équité en matière d'emploi. Les minorités visibles sont désignées comme des «personnes, autres que les autochtones, qui ne sont pas de race blanche ou qui n'ont pas la peau blanche» (Gouvernement du Canada, 1995). Le Groupe de travail interministériel sur l'équité en emploi retient 11 groupes identifiés comme faisant partie d'une «minorité visible» : 1) les Noirs; 2) les Chinois; 3) les Philippins; 4) les Japonais; 5) les Coréens; 6) les Asiatiques du Sud et les Indiens de l'Est; 7) les Asiatiques du Sud-Est; 8) les Asiatiques de l'Ouest non blancs; 9) les Nord-Africains non blancs ou Arabes; 10) les Latino-Américains non blancs et 11) les personnes d'origine mixte, c'està-dire dont l'un des parents provient de l'un des groupes précités. Dans les programmes fédéraux et provinciaux, la notion de minorité ne s'applique qu'aux minorités «visibles». Mais certains programmes provinciaux et fédéraux ont introduit la notion de minorités ethniques ou de communautés culturelles. Statistique Canada souligne que l'origine ethnique ne doit pas être confondue avec la citoyenneté ou la nationalité, qu'elle fait «référence $\mathrm{au}(\mathrm{x})$ groupe(s) ethnique(s) ou culturel(s) auquel (auxquels) appartenaient les ancêtres du répondant. Un ancêtre est une personne dont on descend et qui est habituellement plus éloignée que les grands-parents. L'origine ethnique est liée aux racines ancestrales ou aux antécédents de la population [....]» (Statistique Canada, 2001).

2. Au Québec, les programmes d'accès à l'égalité sont prévus par les articles 86 à 92 de la Charte des droits et libertés de la personne (Bauer, 1994). 
Le moins que l'on puisse dire, c'est qu'une grande confusion règne dans les tentatives de désigner certains groupes-cibles à l'intérieur de l'État-nation canadien. L'absence de cohérence est évidente puisque l'on fait référence à un espace géocontinental (sud-asiatique), à la langue (arabe), à la nationalité (Coréens) ou à la couleur de la peau (Noirs). La notion de minorités visibles est très explicitement empreinte de critères racialisants. La notion de communauté ethnoculturelle reste très ambiguë. La Commission des droits de la personne du Québec (Bauer, 1994) laisse entendre, quant à elle, qu'une communauté ethnoculturelle est composée de personnes originaires de l'Europe.

\section{L'insertion des policiers minoritaires}

Malgré l'introduction de programmes d'accès à l'égalité en emploi, les commissions d'enquête sur les relations entre la police et les minorités racialisées au Canada et au Québec constatent que les groupes minorisés sont encore sous-représentés dans les services de police (Gouvernement de l'Ontario, 1989; Gouvernement du Québec, 1988; 1992). Cette sousreprésentation est imputée autant à des failles dans les stratégies et les pratiques de recrutement des organisations policières qu'aux résistances des communautés minoritaires à faire carrière dans une organisation perçue comme raciste et ce, autant à leur égard qu'à celui des policiers minoritaires. À partir des années 1980, le débat s'est cristallisé sur l'urgence et la nécessité de recruter des représentants des minorités racialisées dans la police de telle sorte que de nombreux travaux et réflexions sont produits sur le thème des politiques, pratiques et stratégies de recrutement des services de police à l'égard des minorités (Jaywardene et Talbot, 1990; Suriya, 1990; 1992; Holdaway, 1991 ; Christidis, 1992).

La difficulté constante à recruter des membres issus de groupes racialisés a contribué à faire converger la recherche vers l'analyse de la sousreprésentation des policiers minorisés (Jaywardene et Talbot, 1990; Stenross et Wilcox, 1990; Doyle, 1991; Holdaway, 1991; Christidis, 1992; Suriya, 1992; Winters, 1992; Kaminski, 1993) ou encore vers des études stratégiques visant à accroître la représentation des groupes racialisés dans les forces de l'ordre (Oscroft, 1991; Tolley et Thomas, 1992). Par contre, l'expérience des policiers minoritaires reste peu documentée. Les données existantes sur la situation des policiers minoritaires font état d'une double marginalisation : marginalisés par leurs collègues «blancs», ces policiers seraient aussi ostracisés par les communautés 
minoritaires (Alex, 1969; Campbell, 1980; Katsuya et Goup, 1994; Benoît, 1998; Jaccoud, 2002). Diverses formes de discrimination et de racisme dont ils seraient l'objet une fois en fonction sont mises en évidence : chances restreintes de promotion, assignations à des tâches spécifiques et à des quartiers à prédominance ethnique, plaisanteries à caractère raciste, insultes, attitudes de suspicion, rejet (Leinen, 1984; Wilson et al., 1984 ; Jaywardene et Talbot, 1990; Benoît, 1998; Holder et al., 2000 ; Jaccoud, 2002; Onifade, 2002). Certains auteurs émettent l'idée que le racisme au sein des corps policiers serait plus subtil actuellement que par le passé (Holdaway, 1996; Jaccoud, 2002). Les formes de résistance et les stratégies d'adaptation du policier minoritaire n'ont pas fait l'objet d'analyses systématiques. On retrouve l'idée que ce policier développe une attitude de négation à l'endroit de son appartenance ethnique (Alex, 1969), utilise des pratiques plus répressives (Leinen, 1984) ou moins répressives (Walker, 1983) à l'endroit des communautés minoritaires, adopte certaines caractéristiques de la culture policière ${ }^{3}$ dont l'humour (Benoît, 1998), voire abandonne la carrière policière (Holdaway, 1993; Onifade, 2002).

Les conséquences et les effets de l'intégration des groupes ethnicisés et racialisés dans les forces de l'ordre, notamment sur le plan des pratiques policières, n'ont guère fait l'objet d'analyse et d'évaluation au Canada. Certains émettent des doutes ou des réserves quant aux effets attendus par les tenants d'une telle politique. Selon certains, la politique de recrutement n'améliorerait pas les rapports entre la police et les minorités ethniques puisque les policiers ethnicisés/racialisés changeraient sous l'influence de la culture organisationnelle et celle des collègues en place. Ils se distancieraient de leur communauté d'origine et perdraient leur attribut ethnique (Jaywardene et Talbot, 1990; Normandeau, 1990; Katsuya et Goup, 1994). Cette perte d'attribut est perçue comme une entrave à la possibilité qu'un policier minoritaire puisse faire le lien avec sa communauté d'origine, diminuant par le fait même le poids des justifications de type intra-organisationnel faisant valoir les avantages politiques et pratiques que l'organisation policière est susceptible de retirer en embauchant un policier issu d'un groupe minoritaire. Par conséquent, la dilution des attributs ethniques pourrait, aux dires de certains, nuire aux effets recherchés par les tenants de la politique d'action positive (Forcese,

3. Culture policière que les travaux britanniques nomment la «canteen culture» (Reiner, 1994). 
1989). Graham (1990) estime qu'il est erroné mais aussi préjudiciable, de penser que les membres de groupes minoritaires deviennent des porteparole de leur groupe : «Cet argument revient en gros à faire du stéréotypage. Il est préjudiciable de prétendre que tous les individus de même couleur, de même religion ou de même sexe représentent leur groupe en pensée et en action» (p. 4). Ajoutons que les groupes minoritaires émettraient eux-mêmes certaines réserves. Ces derniers estiment que le recrutement ne changera pas leur propre perception de la police, qu'il ne contribuera pas à transformer leurs rapports avec la police et ce, en raison de la culture organisationnelle et de la discrimination interne qui réduisent les chances de promotion des policiers minoritaires (Equal Opportunity Consultants, 1992 ; Jaccoud, 2002). Jaywardene et Talbot (1990) critiquent fortement l'argument selon lequel le recrutement de policiers minoritaires contribue à améliorer les relations avec le public, puisqu'ils estiment que la qualité de ces relations dépend davantage du contexte dans lequel la recrue est appelée à exercer sa fonction que de son statut. Brodeur (1991) constate d'ailleurs que dans les pays où une augmentation des représentants des communautés culturelles est réelle, les rapports entre la police et les communautés minoritaires ne se sont pas améliorés.

\section{Problématique et méthodologie de la recherche}

À l'instar de l'enrichissante production théorique sur les concepts d'ethnicisation et de racialisation (voir entre autres Barth, 1969; Weber, 1971; Juteau-Lee, 1983), il convient de rappeler que les notions de race et d'ethnicité sont des construits sociaux, l'ethnicité comportant une part de subjectivité et une part d'objectivité. L'ethnicisation est un processus d'auto-identification et d'auto-attribution à une appartenance culturelle (dans le cours d'un processus de socialisation dans un milieu donné) ou un processus de désignation et de stigmatisation ou encore procède des deux simultanément. Dans ces circonstances, trois cas de figure sont possibles. Je peux : 1) me définir comme minoritaire sans que les autres me désignent comme tel; 2) me définir comme minoritaire et être défini comme tel par les autres; 3 ) ne pas me définir comme minoritaire, mais l'être par les autres. Comme nous le constatons, les effets des politiques d'insertion de policiers minoritaires sont peu connus ou moins bénéfiques qu'attendus. Au Canada et au Québec, peu de recherches se sont intéressées aux expériences vécues par les policiers minoritaires. Or, il 
s'agit certainement d'une exploration incontournable pour qui souhaite déterminer si les objectifs poursuivis par les politiques d'accès des groupes minorisés aux fonctions policières sont atteints. Cette question de recherche reste évidemment complexe puisque ces objectifs, comme nous l'avons souligné, sont eux aussi complexes ${ }^{4}$.

Notre étude est construite sur la prémisse que l'appartenance à une minorité racialisée (minorité culturelle et minorité visible) est susceptible d'influer sur la qualité de l'insertion au sein du service de police et notamment sur la place occupée par les minoritaires une fois insérés dans l'institution. Cette prémisse revient à nous demander dans quelle mesure l'appartenance (revendiquée ou attribuée) à une minorité racialisée est maintenue, renforcée ou déconstruite une fois le policier minoritaire inséré dans l'institution et quels sont les effets de la construction ou de la déconstruction de ces appartenances sur l'insertion des policiers minoritaires.

Des entretiens réalisés auprès de 47 policiers et policières ciblés par le programme d'accès à l'égalité au SPCUM ont été réalisés en vue de faire valoir leur expérience au sein de l'institution. Plus spécifiquement, l'étude consiste à voir dans quelle mesure les policiers minoritaires se sentent intégrés et acceptés dans l'organisation et à évaluer dans quelle mesure les clivages identitaires subsistent, se renforcent, se transforment, s'atténuent ou se fondent dans les interactions qui se tissent au sein de l'organisation policière.

Les 47 policiers désignés par le service de police comme appartenant aux groupes ciblés par les programmes d'accès à l'égalité ont été rencontrés dans le cadre d'entrevues rétrospectives de type semi-directif ${ }^{5}$. La sélection a été établie selon cinq critères de diversification : 1) l'origine ethnique, 2) le genre, 3) la localisation du poste, 4) le grade et 5) la date d'entrée au service.

En fonction de ces critères, notre population est composée de 13 femmes et 34 hommes. Vingt-sept policiers et policières se définissent ou sont définis par le service de police comme appartenant à une minorité visible, 17 à un groupe ethnoculturel (dont 9 sont originaires de pays situés au sud, 5 à

4. Voir Jaccoud et Felices (1999) pour une analyse de la diversité des arguments invoqués pour justifier le recrutement de groupes minoritaires dans les forces de l'ordre.

5. Au moment de la réalisation de ces entretiens (1997-1998), le Service de police de la Communauté urbaine de Montréal (SPCUM) comptait 3956 policiers dont 300 (7,6\%) provenaient de communautés ethnocuturelles, $83(2,1 \%)$ de «minorités visibles» et $9(0,23 \%)$ de communautés autochtones (source : entrevue auprès du responsable du programme d'accès à l'égalité au SPCUM). 
l'est et 3 au nord de l'Europe) et 3 à un groupe d'origine autochtone. Plus de la moitié des répondants sont entrés au service de police dans les années 1990 (55\%); 36\% ont été embauchés dans les années 1980 et $9 \%$ entre 1965 et 1976. Notre échantillon comprend une majorité d'agents (36 répondants), 10 sergents et un commandant. Les répondants ont été sélectionnés dans différents secteurs du territoire desservi par le service de police. Quatre thèmes ont été introduits dans le protocole d'entrevue : le choix de carrière, l'expérience d'insertion au sein du service, les relations avec la communauté et le point de vue sur le Programme d'accès à l'égalité adopté par le Service de police de la Communauté urbaine de Montréal (SPCUM). Dans le cadre de cet article, seuls les résultats entourant le choix de carrière et l'expérience d'insertion sont utilisés.

\section{Le choix de carrière}

L'introduction du choix de carrière comme thème d'entrevue visait à déterminer non seulement les raisons motivant le choix d'opter pour le travail policier, mais aussi les réactions de l'entourage en vue d'examiner dans quelle mesure le métier de policier constitue ou non une rupture culturelle, sociale ou professionnelle par rapport au milieu d'origine.

L'analyse des récits permet de constater que les motivations se structurent autour de trois grands types. Le premier, le plus souvent cité par les répondants, est d'ordre financier. Les répondants considèrent que la carrière policière assure un salaire appréciable et offre une stabilité d'emploi. Le deuxième type de motivation est intrinsèque à l'activité policière ou aux représentations qu'ont les policiers de la nature de cet emploi. Parmi ces représentations, on retrouve l'intérêt pour un métier décrit comme procurant $\mathrm{du}$ «pouvoir», un métier perçu comme «non routinier et plein d'action», qui s'exerce à l'extérieur d'un bureau et dans lequel l'aide apportée à autrui est importante. Le troisième type de motivation, plus rarement exprimé, est d'ordre politique : certains répondants affirment avoir été motivés par une volonté de changer l'organisation policière, d'améliorer les relations avec les minorités ethniques, de transformer l'image de la police auprès des communautés ethnoculturelles, mais aussi par le souci de modifier les stéréotypes des policiers à l'égard de milieux racialisés ou socio-économiquement marginalisés. Il est particulièrement intéressant, et sans doute révélateur, de constater que ce troisième type de motivation est mis de l'avant, à une exception près (un répondant d'origine italienne), par des policiers appartenant à des minorités racialisées 
(principalement Noirs et Asiatiques). Certains événements conflictuels dans lesquels la police et les minorités ont été impliquées (c'est le cas notamment de «l'affaire Griffin-Gosset ${ }^{6}$ » à Montréal) ont eu des effets différenciés sur le choix de carrière : pour certains répondants, ces événements ont eu un effet propulsant dans le choix de carrière alors que pour d'autres, ils ont retardé la décision de faire carrière dans la police.

\section{La réaction de la parenté et de l'entourage}

La majorité des répondants ont dû faire face à des résistances de la part de leur famille immédiate (parents) ou de leur entourage (parenté éloignée et amis) au moment de leur décision de faire carrière dans la police. Plusieurs raisons semblent amener parents et amis à s'opposer ou tout au moins à désapprouver le choix de carrière du répondant : image dépréciée de la police, image le plus souvent constituée à partir de l'expérience vécue dans le pays d'origine de la famille; incompatibilité attribuée de la carrière policière avec les traditions et les valeurs de la famille, le métier de policier étant perçu comme une menace aux valeurs et traditions familiales (cas notamment du rôle que certains milieux familiaux attribuent à la femme). La carrière policière est dépréciée également par certains milieux familiaux qui privilégient l'éducation et les professions libérales. Certains répondants ont vécu des rapports conflictuels avec leur milieu familial en raison de la rupture qu'occasionnait leur choix de carrière avec les traditions professionnelles du milieu familial d'origine. Finalement, plusieurs répondants ont vécu des formes de résistance de la part de leur milieu parental en raison des dangers associés à l'exercice de cette profession. Les encouragements de la part des familles et de l'entourage sont exceptionnels. En fait, plus les valeurs des milieux familiaux sont décrites par les répondants comme similaires ou proches des valeurs du groupe majoritaire (les Québécois) et plus le milieu familial constitue un soutien pour l'aspirant à une carrière policière. Par contre, dans certains cas, la fragilité du statut d'immigrant incite certains parents à apporter leur soutien, le service de police étant perçu comme assurant une sécurité économique. L'insertion socioéconomique de l'immigrant devient, dans ces conditions, plus importante que le respect des traditions et des valeurs familiales.

6. Anthony Griffin, un jeune noir, est abattu en 1987 par le policier Allen Gosset, alors qu'il tente de s'échapper du stationnement d'un poste de police. 


\section{Les expériences d'insertion au service de police}

Les entretiens ont été menés de manière rétrospective, afin de mieux saisir l'évolution de l'expérience d'insertion des policiers au sein du service. Les événements problématiques vécus par les répondants ont été analysés à partir de critères tels que leur durée (quelques mois, plusieurs années, incessants), leur constance ou leur fréquence (événements sporadiques et isolés ou événements répétés et continus) et leur nature (commentaires, gestes, atteintes à l'intégrité physique et/ou morale des individus). L'interprétation des événements a soulevé l'épineuse question du décalage entre l'objectivation et la subjectivation des événements. En effet, certains policiers font état de difficultés objectives qu'ils ne considèrent pas toutefois comme subjectivement problématiques. Dans ces circonstances, l'interprétation du répondant a constitué le critère décisif de la qualification de l'expérience d'insertion.

L'analyse des 47 récits d'expérience permet d'entrevoir que la majorité des répondants affirment avoir vécu une excellente ou une bonne intégration dans le service (31 répondants, soit $68 \%$ de notre échantillon). Parmi les répondants ayant relaté et interprété leur insertion au service comme étant difficile, voire très difficile (16 répondants, soit $32 \%$ de notre échantillon), la majorité (75\%) est identifiée à une minorité visible, alors que les minorités visibles ne constituent que $48 \%$ des répondants affirmant avoir vécu une bonne insertion au service de police. Les répondants appartenant à un groupe ethnoculturel semblent plus susceptibles de vivre une meilleure insertion au service de police que les répondants identifiés à une minorité visible. L'appartenance à une catégorie de genre semble moins significative que l'appartenance à une catégorie «ethnique» pour différencier les expériences positives et négatives d'insertion. Cependant, les répondants ayant relaté des difficultés dans leur insertion au service de police comptent proportionnellement davantage de femmes que dans le groupe des répondants ayant affirmé avoir vécu une bonne, voire une excellente intégration au service de police $(31 \%$ de femmes dans le premier groupe contre $26 \%$ dans le second). Les tableaux 1 et 2 présentent de manière plus détaillée les profils de genre et d'ethnicité des répondants en fonction de la qualité de l'insertion dans le service.

\subsection{Les bonnes et excellentes expériences d'intégration}

Les policiers qui s'insèrent dans cette catégorie disent n'avoir vécu aucune difficulté dans leur processus d'intégration ou des difficultés qu'ils qua- 
TABLEA U 1

Expériences d'insertion selon le genre

\begin{tabular}{|l|c|c|c|}
\hline \multirow{2}{*}{ Genre } & \multicolumn{2}{|c|}{ Expériences d'insertion } & \multirow{2}{*}{ Total } \\
\cline { 2 - 3 } & Très difficile/difficile & Excellente/bonne & 34 \\
\hline Hommes & $11(69 \%)$ & $23(74 \%)$ & 13 \\
\hline Femmes & $5(31 \%)$ & $8(26 \%)$ & 47 \\
\hline Total & $16(100 \%)$ & $31(100 \%)$ & \\
\hline
\end{tabular}

TABLEAU 2

Expériences d'insertion selon l'origine

\begin{tabular}{|l|c|c|c|}
\hline \multirow{2}{*}{ Origine } & \multicolumn{2}{|c|}{ Expériences d'insertion } & \multirow{2}{*}{ Total } \\
\cline { 2 - 3 } & Très difficile/difficile & Excellente/bonne & 14 \\
\hline Noirs & $5(31,25 \%)$ & $9(29 \%)$ & 6 \\
\hline Asiatiques & $3(18,75 \%)$ & $3(9,7 \%)$ & 4 \\
\hline Arabes & $2(12,5 \%)$ & $2(6,4 \%)$ & 3 \\
\hline Latino-Américains & $2(12,5 \%)$ & $1(3,2 \%)$ & $\mathbf{2 7}$ \\
\hline Groupes ethnoculturels & $\mathbf{1 2}(\mathbf{7 5} \%)$ & $\mathbf{1 5}(\mathbf{4 8} \%)$ & $\mathbf{1 7}$ \\
\hline Autochtones & $\mathbf{4}(\mathbf{2 5} \%)$ & $\mathbf{1 3}(\mathbf{4 2} \%)$ & 3 \\
\hline Total & $0(0 \%)$ & $3(9,7 \%)$ & $\mathbf{4 7}$ \\
\hline
\end{tabular}

lifient de mineures ou dont ils déconstruisent toute intentionnalité raciste. Généralement, les policiers affirment que les remarques dont ils font l'objet sont des blagues typiques du milieu policier. Ces remarques sont au contraire interprétées comme une marque d'acceptation par le milieu à leur endroit. Pour illustrer ce genre d'attitude, nous pouvons citer l'exemple d'un policier d'origine asiatique :

Ah oui, c'est... Y [en a pour] taquiner par rapport à votre peau, à votre race. $Y$ font des blagues qu'y feraient pas peut-être à un autre Québécois, y pourraient pas le faire, les circonstances adonnent pas, tsé... Fait que (rires) aussi ben... Tsé, on le sait quand c'est une blague. C'est pas une question de racisme, on le sait quand c'est une blague. Moi aussi j'en fais des blagues, que ça soit sur les Blancs ou sur les Noirs. Mais quand c'est une blague c'est une blague (\# 33, policier d'origine asiatique, agent). 
En général, les remarques adressées par les autres policiers concernent les difficultés d'expression, l'âge (les postulants visés par le programme d'accès à l'égalité du service de police étant habituellement plus âgés), les habitudes alimentaires, la «race», la couleur de peau :

Ben, ça peut être la race, la couleur de peau. Ça peut être... la grandeur aussi.

Moi je suis pas très grand, je suis pas très gros. Ça aussi ça peut [jouer] (\# 33, policier d'origine asiatique, agent).

Les difficultés exprimées par ces policiers sont associées à des incidents spécifiques et très sporadiques dans le temps; elles sont tolérées lorsqu'elles ne remettent pas en question leur compétence ou lorsqu'elles sont exprimées par des collègues avec qui l'habitude de travailler s'est installée :

Mais moi, quand ça venait de lui [un supérieur], je prenais ça comme... Ça m'affectait pas... personnellement.... du moment où au niveau du travail je sais que ça va pas l'influencer, alors là ça me mettait ouvert à tout commentaire. Dans le sens que même si il faisait des blagues sur les Noirs, tout ça, ça ne me dérangeait pas du tout, parce que je savais pertinemment bien que mon travail que je faisais, il l'appréciait.... ça dépend avec qui. C'est ça. Oui, quelqu'un que je connais pas ou que je travaille (pour la première fois) pis qui va me faire une blague, ça je l'accepte pas. Mais un collègue avec qui je travaille, ça ça me dérangeait pas (\# 12, policier noir, agent).

Nombreux sont les répondants qui, dans ce sous-groupe, adoptent la «culture policière» en s'appropriant les blagues à l'endroit des minorités racialisées, une manière, dit-on, de «faire tomber la tension raciale»:

Je leur ai dit : je vais les faire, mais la condition c'est seulement moi ici qui a le droit de faire des jokes sur les Noirs... Et le monde a ri. Je leur donnais les jokes et je leur ai dit que moi avant ça j'étais un Blanc. Tout le monde se retourne pour comprendre la suite, puis je leur dis qu'il y avait un concours de bronzage dans mon quartier, et j'ai gagné. Je suis resté comme ça. Et puis ils sont contents, on riait. Et puis la tension venait de tomber. La tension raciale venait de tomber. Vraiment tomber (\# 47, policier noir, agent).

À tel point que l'intégration est vécue comme une marque de «blanchitude» : «Je n'étais plus un noir à c't heure. Non. J'étais devenu un collègue» (\# 47, policier noir, agent).

De manière générale, les policiers issus des groupes ethnoculturels analysent la qualité de leur intégration par leur non-appartenance à une minorité genrée (cas des hommes) ou racialisée. D'ailleurs, de nombreux répondants se sont montrés outrés d'avoir été contactés par l'équipe de recherche sur la base de leur inscription à la liste du programme d'accès à l'égalité du service de police, ignorant y figurer. Les répondants estiment 
que leur appartenance au groupe majoritaire (adhésion aux «valeurs culturelles québécoise») ou, parallèlement, leur non-appartenance à la culture d'origine ou, devrait-on dire, leur non-appartenance à la culture marquant leur inscription dans la liste du PAE, ont facilité leur insertion au service. Les qualités personnelles (communication, autonomie, compétence) sont également mentionnées comme critères facilitant l'intégration. Quelques policiers, en particulier ceux qui ont été recrutés récemment, considèrent que l'intégration des minorités ne constitue plus un problème dans un service de police décrit comme ouvert et accoutumé à la diversité culturelle.

\subsection{Les expériences difficiles et très difficiles}

Une des caractéristiques des récits des 16 policiers ayant relaté avoir vécu ou vivant encore des difficultés à s'insérer au SPCUM est le fait que la période présentée comme étant la plus pénible est celle de la période probatoire. Par définition, la période probatoire est une période instable durant laquelle le policier vit de nombreuses pressions : il doit confirmer une compétence présumée par son succès au processus de sélection, il est en période d'apprentissage, il est soumis à une certaine observation et un certain contrôle. Compte tenu de son statut, le policier en période probatoire dispose évidemment de conditions moins favorables. Il est dans un état de dépendance qui le muselle en quelque sorte; l'organisation de la période probatoire ne permet pas au policier de changer de poste. Sa marge de manœuvre s'en trouve par conséquent réduite et il ne peut avoir recours à l'une des stratégies de résolution des problèmes dans un poste : la mutation.

Les expériences d'intégration difficiles varient en ce qui a trait à leur durée, leur fréquence et leur nature. Trois facteurs, qui se combinent, sont particulièrement décisifs dans la répartition de cette diversité : le statut de genre, le statut ethnique et la représentativité ethnique au sein du service ou du poste à l'arrivée. Aussi, les femmes et les hommes appartenant à des minorités visibles (en particulier les Noirs et les Asiatiques) et les minoritaires pour qui la représentativité de leur groupe d'appartenance ethnique était quasi nulle à leur entrée en fonction sont les personnes ayant éprouvé le plus de problèmes d'insertion et ce, sur le plan de la durée, de la fréquence et de la nature des événements. Certains policiers disent avoir vécu de nombreux problèmes surtout au début de leur carrière (les 3-4 premiers mois ou la première année, qui correspond à 
l'année de la période probatoire), d'autres parlent des premières années (3 à 4 ans), alors que quelques policiers parlent de constance tout en précisant que la fréquence des problèmes a diminué.

Le genre de problème auquel les policiers ont dû faire face de la part des collègues ou des supérieurs, ou le genre de situation défini comme problématique pour ces policiers, est varié. Les problèmes les plus fréquemment cités sont les commentaires sur les liens entre l'origine et la facilité d'embauche (par exemple, les policiers minoritaires entendent sur une base régulière qu'ils sont des «voleurs de job» ou que les critères d'embauche ont été abaissés dans leur cas ou qu'ils ont bénéficié de passe-droits ou encore que les promotions leur sont plus accessibles), ainsi que les blagues à connotation racialisante, blagues que le jargon policier nomme le «tirage de pipe» (par exemple un policier d'origine italienne est surnommé «le Mafioso», un policier d'origine arabe «le conducteur de chameau»). Les répondants de ce sous-groupe sont également confrontés à des commentaires sur leur formation académique, considérée comme incompatible avec la fonction policière; ils font état de commentaires émis sur leur physique (petite taille, manque de force, couleur de peau) ou sur leur manière de s'exprimer (par exemple, une policière italienne anglophone se fait dire de se «faire checker les oreilles»). Ils relatent avoir vécu des démonstrations ouvertes d'incompréhension à l'endroit des habitudes culturelles (par exemple, on commente négativement l'emprise de la famille ou on affiche une attitude de dégoût devant un repas qu'un répondant mange). Les doutes émis sur leur compétence sont parfois énoncés. Quelques répondants ont vécu des affronts de la part de leur collègue devant les citoyens. L'isolement et le manque de soutien font également partie des difficultés relatées. Certains répondants estiment qu'ils ont fait l'objet de surveillance accrue en raison d'attitudes de suspicion sur leur qualification ou sur leur allégeance à l'institution. D'autres relatent avoir été restreints dans leur mobilité professionnelle, notamment en raison de refus essuyés dans les affectations (postes et fonctions). Il est arrivé fréquemment que les répondants s'insurgent contre l'utilisation médiatique dont ils ont été l'objet par le service de police au moment de leur embauche. L'assignation à des tâches différentielles (travail de bureau, assignation à la rédaction des rapports, refus de laisser conduire l'auto-patrouille) ou à des quartiers spécifiques (quartiers à forte concentration ethnique) est vécue comme un traitement discriminatoire à leur endroit. Certains ont vécu un rejet très explicite de la part de collègues (policiers qui refusent ou démontrent une 
réticence à travailler avec eux). Des insultes et commentaires dégradants sont parfois utilisés (par exemple se faire traiter de «waps» ou entendre des commentaires tels que «va faire du ciment» ou «mangeur de vidanges»). Quelques policiers ont même été victimes de menaces, de crachats et de méfaits sur leurs biens personnels (notamment une voiture endommagée).

Devant les difficultés, les policiers réagissent différemment et n'utilisent pas nécessairement les mêmes stratégies. Trois types de stratégie et de réaction sont adoptés :

1. Des stratégies de résistance et d'affirmation de soi : ce sont des stratégies qui visent à changer l'institution et ses acteurs; dans ce registre, les attitudes consistent à maintenir ses habitudes en dépit des remarques, à s'autovaloriser, à négocier avec les collègues et à les sensibiliser aux différences. Quelques répondants utilisent la confrontation et la réplique et, exceptionnellement (un seul répondant dans notre échantillon), le processus de plainte est adopté.

2. Des stratégies de résignation, de retrait ou de fuite : ces stratégies constituent des formes d'accommodement du policier à l'institution. Changer de poste, de fonction ou de partenaire, espérer l'ancienneté pour se faire respecter, se taire ou se détacher de son milieu de travail en érigeant une frontière stricte entre vie privée et vie professionnelle sont les principales actions et attitudes adoptées dans ce registre. Exceptionnellement, certains répondants ont songé à abandonner la carrière policière.

\section{Des stratégies actives de conformité à l'institution ou aux} acteurs du groupe majoritaire : ces stratégies visent à s'autotransformer pour devenir conforme au milieu dans lequel le policier évolue. Elles font appel à des comportements tels que : accroître son rendement, réinterpréter les commentaires, les banaliser et les accepter comme inhérents à la culture policière ou comme étant constitutifs d'un rite de passage obligé, endosser la responsabilité des problèmes soulevés par les collègues, se distancer de sa communauté d'origine ou avoir recours à un soutien psychologique.

\section{Conclusion}

Les expériences positives d'insertion soulignent que l'appartenance à une minorité racialisée et culturalisée se voit déconstruite, soit par le 
répondant lui-même qui ne se définit pas comme un minoritaire, soit par les autres acteurs qui ne perçoivent plus le répondant comme un minoritaire (situation vécue par des policiers noirs qui ne sont même plus perçus comme des Noirs). Elles soulignent parallèlement que l'appartenance à une minorité racialisée et culturalisée est reconstruite ou maintenue par le répondant et par les autres acteurs sans que la référence identitaire soit problématisée (répondants n'éprouvant aucune difficulté entourant leur statut ou réinterprétant les références identitaires comme non problématiques).

Dans le cas des expériences difficiles d'intégration, nous constatons que le processus d'insertion n'est pas linéaire et ne s'inscrit pas nécessairement dans un processus continu partant d'une marginalisation vers une intégration. Dans certains cas, l'insertion doit être renégociée à chaque changement d'affectation. Les plus grands marqueurs des différenciations sont l'appartenance à une catégorie de genre (homme vs femme), l'appartenance à une catégorie de couleur (blanc vs visible), l'appartenance à une catégorie linguistique (francophone vs anglophone) et l'appartenance à une catégorie éducation-formation (formation universitaire vs non universitaire).

Les résultats de notre étude nous invitent à relativiser la portée des programmes d'accès à l'égalité. D'une part, les frontières identitaires ne sont pas déconstruites et subsistent au sein de l'organisation policière; d'autre part, la diversité incluse dans l'organisation policière est une diversité de surface puisque, dans certains cas, le service intègre des minoritaires qui, en fait, ne se perçoivent pas comme tels. L'inclusion d'une diversité virtuelle présuppose que les principes et les pratiques de sélection imposés aux candidats avant leur entrée dans l'organisation visent à neutraliser des caractéristiques qui seraient considérées comme incompatibles ou entrant en conflit avec la culture organisationnelle. Elle laisse entendre également que le policier minoritaire est instrumentalisé dans la mesure où son apparence physique ou ses connaissances linguistiques sont utilisées pour satisfaire les fonctions opérationnelles du service tout en préservant les valeurs, les principes et l'idéologie de l'organisation. Elle souligne également que les politiques d'inclusion des groupes minoritaires dans les services de police consistent, en partie, à intégrer des attributs «visibles» ou «audibles» pour remplir des fonctions politiques indéniables (maintien d'une image publique d'un service représentatif de la diversité culturelle et conformité aux exigences légales). L'organisation policière accepte, en son sein, ou des individus préalablement conformes à la culture organi- 
sationnelle, ou des individus moins conformes qui sont transformés sous le poids des pressions, ou encore des individus qui sont maintenus en marge de l'institution. Dans ces circonstances, il convient de conclure que l'un des objectifs recherchés par les programmes d'accès à l'égalité, notamment celui d'inclure la diversité en espérant transformer les pratiques organisationnelles et, ce faisant, améliorer les rapports avec les communautés dites ethnoculturelles, reste limité.

\section{Références}

Alex, N. (1969). Black in blue : a study of negro policemen. New York : Century Crofts. Barth, F. (1969). Ethnic groups and boundaries: the social organisation of cultural difference. Boston : Little, Brown and Company.

Bauer, J. (1994). Les minorités au Québec. Montréal : Collection Boréal Express.

Benoit, E. (1998). Les policiers et policières noir-e-s d'origine haitienne : étude exploratoire sur leurs pratiques de travail. Mémoire de maîtrise, Département de criminologie, Université d'Ottawa.

Brodeur, J.-P. (1991). Access to justice and equality of treatment. Montréal : Centre international de criminologie comparée.

Campbell, V. (1980). Double marginality of black policemen. Criminology, 17 (4), 477-484.

Christidis, A. (1992). Royal Canadian Mounted Police: strategies in the recruitment of visible minorities. Ottawa : RCMP.

Commission des droits de la personne (1994). Recommandations relatives au questionnaire d'auto-identification des membres des groupes cibles, dans le cadre d'un programme d'accès à l'égalité. In J. Bauer (ed.), Les minorités au Québec (49). Montréal : Collection Boréal Express.

Doyle, C. (1991). Police recruitment from ethnic minorities - a strategy. Londres : Police Requirements Support Unit, Home Office.

Equal Opportunity Consultants (1992). A strategy to enhance the Metropolitan Toronto Police Force's profile with racial minorities. Toronto : Equal Opportunity Consultants.

Forcese, D. (1989). Canada 2000 : race relations and public policy. In O.P. Dwivedi, R. D'Costa, C.L. Stanford \& E. Tepper (eds), Conference on Canadd's race relations options (260-263). Guelph : Department of Political Studies.

Gouvernement de l'Ontario (1989). The report of the race relations and policing task force. Ontario : Department of the Solicitor General.

Gouvernement du Canada (1995). Loi sur l'équité en matière d'emploi, ch. 44.

Gouvernement du Québec (1988). Enquêtes sur les relations entre les corps policiers et les minorités visibles et ethniques: rapport final. Québec : Commission des droits de la personne du Québec (rapport Bellemare).

Gouvernement du Québec (1992). Une occasion d'avancer : rapport sur les relations entre les communautés noires et le service de police de la Communauté urbaine de Montréal. Québec : ministère de la Sécurité publique (rapport Corbo). 
Graham, C.D. (1990). L'action positive dans les services de police : une volonté politique ou morale? Ottawa : Department of the Solicitor General of Canada.

Haveman, P. (1988). The indigenization of social control in Canada. In B.W. Morse \& G.R. Noodman (eds), Indigenous law and the state (71-100). Dordredit : Foris Publication.

Holdaway, S. (1991). Race relations and police recruitment. British Journal of Criminology, 31 (4), 363-382.

Holdaway, S. (1993). The resignation of black and asian officers from the police service. A Report to the Home Office. London : Home Office.

Holdaway, S. (1996). The racialisation of british policing. Londres : McMillan Press.

Jaccoud, M., \& Felices, M. (1999). Ethnicization of canadian policing. Revue canadienne Droit et Société, 14 (1), 83-100.

Jaccoud, M. (2002). Relationships between police and ethnic minorities in Sheffield (U.K). Montréal : Cahier du Centre international de criminologie comparée.

Jaywardene, C.H.S., \& Talbot, C.K. (1990). Police recruitment of ethnic minorities. Ottawa : Canadian Police College.

Juteau-Lee, D. (1983). La production de l'ethnicité ou la part réelle de l'idéal. Sociologie et Sociétés, $X V(2), 39-54$.

Holder, K.A., Nee, C., \& Ellis, T. (2000). Triple jeopardy? Black and asian women police officers' experiences of discrimination. International Journal of Police Science and Management, 3 (1), 68-87.

Kaminski, R.J. (1993). Police minority recruitment : predicting who will say yes to an offer for a job as a cop. Journal of Criminal Justice, 21 (4), 395-409.

Katsuya, L., \& Goup, D. (1994). The Royal Canadian Mounted Police : organizational culture and issues : diversity in ethnic representation : public image. Ottawa : Canadian College of Police.

Leinen, S. (1984). Black police, white society. New York : New York University Press.

Leman, M. (1994). Le multiculturalisme canadien. Bulletin d'actualité. Ottawa : Service de recherche, Bibliothèque du Parlement, Division des affaires publiques et sociales.

Normandeau, A. (1990). La police et les minorités ethniques. Journal du Collège canadien de police, 14 (3), 231-246.

Onifade, D. (2002). The experience of black/minority ethnic police officers, support staff, special constables and resigners. Edinburgh : The Stationery Office Ltd.

Oscroft, G.J. (1991). Policing and integrated racial equality : a study of the dutch experience. Positive action initiatives in recruitment and trainning. Nottingham : Nottinghamshire Constabulary.

Reiner, R. (1994). Policing and the Police. In M. Maguire, R. Morgan \& R.Reiner (eds), The Oxford handbook of criminology (705-772). Oxford : Clarendon Press.

SPCUM (1997). Rapport du programme d'accès à l'égalité. Service de police de la Communauté urbaine de Montréal, document inédit.

Statistique Canada (2001). Recencensement de la population 2001. http://www12.statcan.ca/francais/census01/Products/Analytic/companion/etoimm/def_f.cfm. 
Stenross, B., \& Wilcox, S.P. (1990). Do preemployment tests always hurt minorities? The case of nonwhite employment in law enforcement. Sociological Practice Review, 1 (2), 105-108.

Suriya, S.K., (1990). Toward employment equity : the representation of visible minorities in Canadian police forces. A public policy review. Ottawa : Carleton University, Department of Geography.

Suriya, S.K. (1992). The representation of visible minorities in canadian police : employment equity beyond rhetoric. Los Angeles : University of Southern California.

Tolley, H., \& Thomas, K. (1992). Police access trainning. A case study of positive action and ethnic minority recruitment to the West Midlands Police Force. Londres : Commission for Racial Equality.

Walker, D.B. (1983). Black police values and the black community. Police Studies, 5 (4), 20-28.

Weber, M. (1971). Économie et Société. Paris : Plon.

Wilson, D., Holdaway, S., \& Spencer, C. (1984). Black police in the United Kingdom. Policing, 1 (1), 20-30.

Winters, C.A. (1992). Socio-economic status, test bias and the selection of police. Police Journal, 65 (2), 125-135. 